\title{
Identification of interfacial defects in the layered structure of a chalcopyrite compound
}

Guangming Cheng and Nan Yao

Princeton Institute for the Science and Technology of Materials, Princeton University, Princeton, New Jersey, United States

Email: gcheng2@princeton.edu and nyao@princeton.edu

CuInS2 is a diamond-like semiconductor with the crystal structure of chalcopyrite that was first synthesized to explore the applicability to ternary compounds of the Grimm-Sommerfeld valence rules for tetrahedral coordination.[1,2] The chalcopyrites have the diamond-like zincblende structure but with two cations ordered on sublattices. The Bravais lattice of the chalcopyrite is body-centered tetragonal, belonging to space group I $\overline{42} \mathrm{~d}$. The direct energy band gap of CuInS2 makes it a strong light absorber, with a value of $1.53 \mathrm{eV}$ that lies at the optimum for efficient single-junction solar cells. Therefore, $\mathrm{CuInS} 2$ has been explored for polycrystalline thin-film solar cells, beginning with a $3.6 \%$ efficient homojunction cell, then $n-C d S / p-$ CuInS2 heterojunction solar cells first reaching 7.3\%,12 and later 11.4\% power conversion efficiency, but far less than the theoretically achievable value of $\sim 30 \%$. The cause of this low performance is not understood. Here, a single crystal grown from $1 \mathrm{~mol} \% \mathrm{Cu}$-deficient melt was studied by using atomic resolution highangle annular dark-field (HAADF) scanning transmission electron microscopy (STEM) and electron dispersive spectroscopy (EDS) (no beam damage issue). [3, 4] Figure 1 shows that the CuInS2 crystal has a lamellar structure, taken in the $\langle 1 \overline{10}\rangle$ direction. The lamellae are alternating pseudotwins that form a herringbone pattern (Fig. 1a,b). The thickness of the lamellae ranges from several nanometers to hundreds of nanometers. The pseudotwin plane was identified as $\{112\}$. The pseudotwins are separated by a rotationaltwin boundary (a $180^{\circ}$ rotation) that has been theorized to be the most energetically favored twin boundary in chalcopyrites, as it avoids the reconstruction that would be inevitable in anion- and cation-terminated twin boundaries (Fig. 1c). Periodic interfacial structures ("interphases") are seen to be inserted into the pseudotwin boundaries. The interfacial structure includes two bright atomic planes but with interplanar spacings different from those of the chalcopyrite CuInS2 bulk. We used STEM-EDS to determine by how much the composition of the interphases deviates from bulk CuInS2 along the trace marked in the HAADF image of Fig. 2. With respect to the bulk, the interfacial layers are depleted of $\mathrm{Cu}$ (down -7.9 at. \% in the single layer and down -11.5 at. $\%$ in the double layer) and contain up to +8.6 at. $\%$ excess In and +3.0 at. $\%$ excess $\mathrm{S}$. Thus, while the bulk contains 25.2 at. $\% \mathrm{Cu}$, the single and double interfacial phase layers contain only 17.1 and 13.7 at. $\% \mathrm{Cu}$, respectively, but 32.9 at. \% In. The copper deficiency of the interfacial phase suggests that the interfacial phase accommodates the copper deficiency of the overall crystal. [5] 


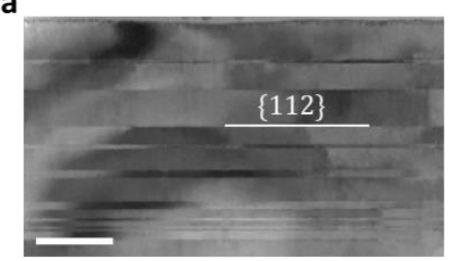

b

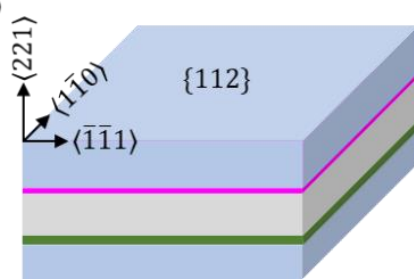

C

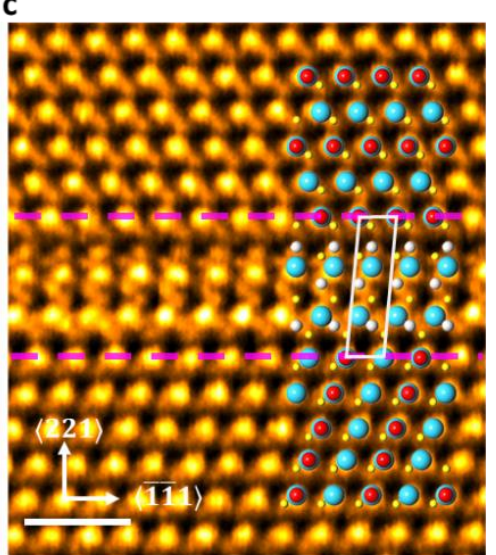

Figure 1. (a) Bright-field TEM image showing lamellar pseudotwins. The pseudotwin plane is $\{112\}$. Scale bar: $500 \mathrm{~nm}$. (b) Schematic illustration of (blue or gray) bulk CuInS2 pseudotwin lamellae separated by a single (magenta) and a double (green) interphase, all stacked in the $\langle 221\rangle$ direction. (c) Atomic-resolution STEM-HAADF images showing parts of chalcopyrite CuInS2 pseudotwins above and below single (top frames) and double layers (bottom), viewed from the $\langle 1 \overline{10}\rangle$ direction. A color-coded chalcopyrite structure $(\mathrm{Cu}$, red; In, blue; S, yellow) is overlaid on the image to identify atoms. In (c), atomic-column pairs of $\mathrm{Cu}$ and In can be seen --- note the alternation of bright (In) and faint $(\mathrm{Cu})$ pairs in the bulk crystal. Scale bar: $1 \mathrm{~nm}$.
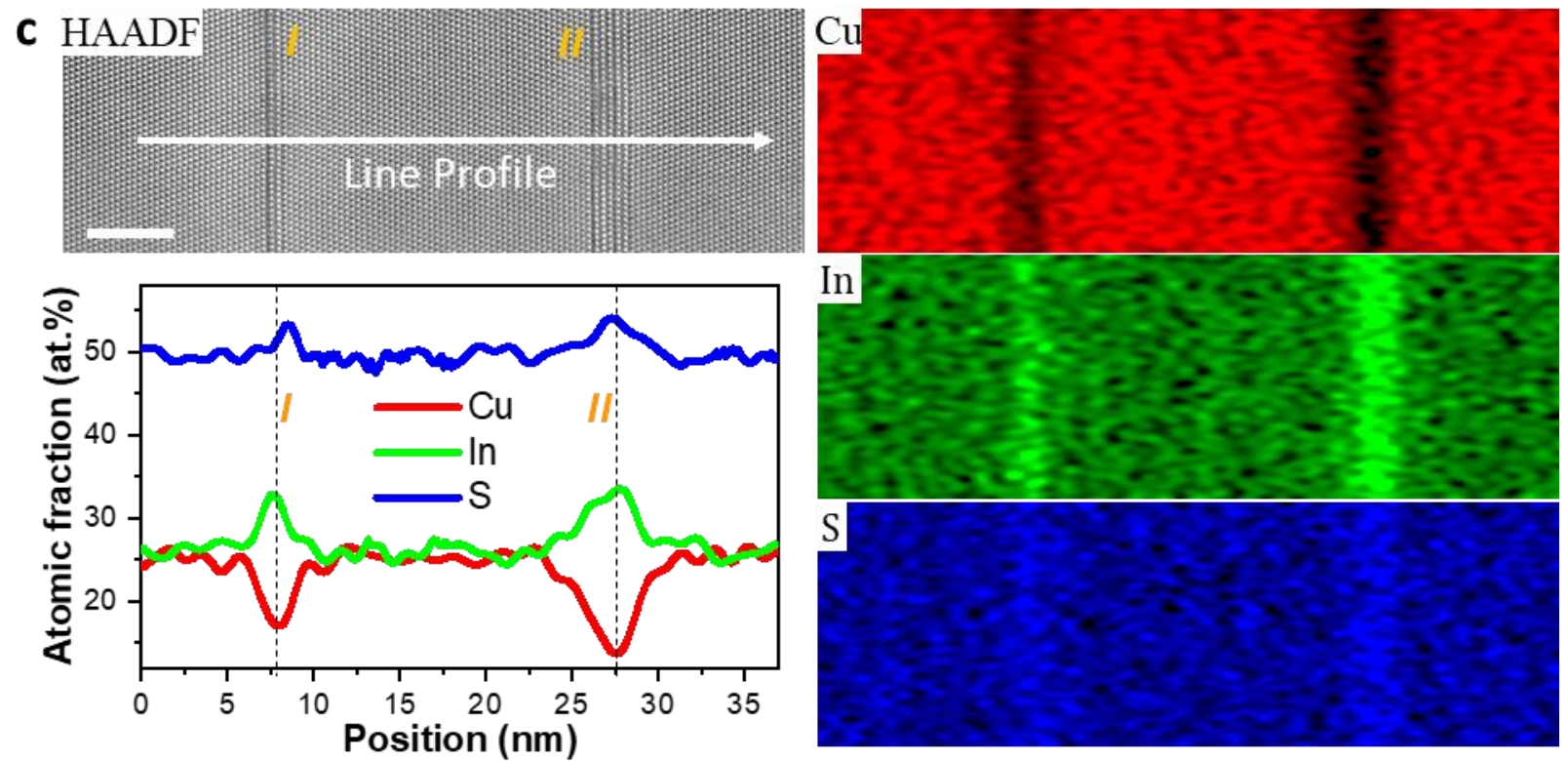

Figure 2. STEM-EDS mapping of bulk CuInS2 including one pseudotwin boundary with a single (I) and one with a double (II) interphase layer. The HAADF image shows the EDS trace across the bulk and the two interphases. The right panel combines the elemental maps of $\mathrm{Cu}$ (red), In (green), and $\mathrm{S}$ (blue) from multiple scans. The compositional profiles along a single scan, at the bottom left, show $\mathrm{Cu}$ depletion and $\mathrm{In}$ and $\mathrm{S}$ enrichment in the interfacial layers. Scale bar: $5 \mathrm{~nm}$. 
[1] B. Tell, J. Shay, H. Kasper, Room-temperature electrical properties of ten I-III-VI2 semiconductors, Journal of Applied Physics 43(5) (1972) 2469-2470.

[2] J.J. Frick, S.K. Kushwaha, R.J. Cava, A.B. Bocarsly, Characterization of Primary Carrier Transport Properties of the Light-Harvesting Chalcopyrite Semiconductors CuIn (S1-x Se x) 2, The Journal of Physical Chemistry C 121(32) (2017) 17046-17052.

[3] J.J. Frick, G. Cheng, S. Kushwaha, N. Yao, S. Wagner, A.B. Bocarsly, R.J. Cava, Observation of [VCu1In i 2+ VCu1-] Defect Triplets in Cu-Deficient CuInS2, The Journal of Physical Chemistry C 124(48) (2020) 26415-26427.

[4] G. Cheng, S. Yao, X. Sang, B. Hao, D. Zhang, Y.K. Yap, Y. Zhu, Evolution of Irradiation-Induced Vacancy Defects in Boron Nitride Nanotubes, Small 12(6) (2016) 818-824.

[5] The authors acknowledge the use of Princeton's Imaging and Analysis Center (IAC), which is partially supported by the Princeton Center for Complex Materials (PCCM), a National Science Foundation (NSF) Materials Research Science and Engineering Center (MRSEC; DMR-2011750). 